\title{
Cochlear function in patients with chronic kidney disease
}

\author{
${ }^{1}$ Discipline of Audiology, University of KwaZulu-Natal, Durban, South Africa \\ ${ }^{2}$ Discipline of Statistics and Computer Science, University of KwaZulu-Natal, Durban, South Africa
}

S M Govender, ${ }^{1}$ B Comm Path (Aud), M Comm Path (Aud); C D Govender, ${ }^{1}$ BA, DOT, M Audiology, MA; G Matthews, ${ }^{2}$ PhD

Corresponding author: S M Govender(munsamys@ukzn.ac.za)

Objective. To evaluate cochlear functioning in patients (18 - 45 years old) with varying stages of chronic kidney disease (CKD). Using purposive sampling, 50 participants, 10 in each of the 5 stages of $\mathrm{CKD}$, were selected and underwent pure tone audiometric testing and distortion product otoacoustic emissions (DPOAEs).

Results. Significant differences $(p<0.05)$ were found between pure tone audiometry and DPOAEs in detecting early cochlear dysfunction in the high-frequency range in stages $3(6000 / 5000 \mathrm{~Hz} ; p=0.00), 4(6000 / 5000 \mathrm{~Hz} ; p<0.03)$ and $5(4000 / 3333 \mathrm{~Hz} ; p<0.01,8000 / 6667 \mathrm{~Hz}$ : $p<0.05)$ with DPOAEs being more sensitive in identifying early cochlear dysfunction. Patients in stages 1 and 2 presented with normal puretone thresholds and DPOAEs, suggesting that cochlear functioning in these patients was normal. Early cochlear dysfunction, thereby indicating a subclinical hearing loss, was identified in stages 3, 4 and 5 by DPOAE testing. In addition, blood test results, drug intake and concomitant conditions were recorded and analysed which suggested a relationship between reduced cochlear functioning and increased electrolyte levels, treatment regimens and concomitant conditions.

Conclusion. Participants in the later stages of CKD presented with early cochlear dysfunction, presenting with subclinical hearing loss. It was postulated that this subclinical hearing loss resulted from a combination of electrolytic, urea and creatinine imbalances, together with concomitant medical conditions and ototoxic drug intake. It was concluded that audiological monitoring be included in the management of patients with CKD and that DPOAEs be introduced as part of the test battery to monitor cochlear function in patients with varying degrees of CKD.

Keywords: cochlea, hearing loss, glomerular filtration, dialysis, pure tone audiometry, otoacoustic emissions, subclinical hearing loss, monitoring, electrolytes

S Afr J CD 2013;60:44-49.DOI:10.7196/SAJCD.243

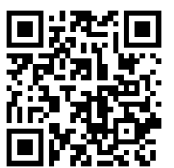

Chronic kidney disease (CKD) is considered a major public health concern because of the increase in prevalence in both adults and children. Unhealthy lifestyles, genetic predisposition, associated health conditions and treatment regimens all contribute to the increasing prevalence of CKD. According to the National Kidney Foundation (NKF) (2003), almost 10\% of all people globally experience some form of kidney disease, with the majority residing within sub-Saharan Africa. This high prevalence, particularly in South Africa, is due to the increasing prevalence of hypertension and diabetes. There are many complications associated with the disease which result in decreased quality of life. One such complication is auditory dysfunction.

Auditory system complications in patients with CKD are multifactorial, arising as a result of ototoxic effects of treatment regimens associated with $\mathrm{CKD}$, and concomitant conditions such as diabetes mellitus, hypertension and lupus, as well as the pathophysiology of the disease itself. Research pertaining to CKD and hearing loss is scarce, particularly within the South African context; however, international literature indicates that the cochlea appears to be the most affected part of the auditory system in patients with CKD (Sobh, Koussi \& Bakr, 1999; Thodi et al., 2006). This is due to the relationship between the cochlea and the kidney, making the physiology of the cochlea dependent upon normal kidney function.

The kidneys and the cochlea have similar physiological mechanisms, including the active transportation of fluids and electrolytes that is performed by the glomerulus of the kidney and the stria vascularis of the ear, respectively (Boys Town National Research Hospital, 1989). The ears and the kidneys develop during the same gestational period (5th to 8th week of pregnancy) (Boys Town National Research Hospital, 1989). When viewed microscopically, the cochlea and the kidneys share similar types of membranes. The arrangement, structural organisation and function of the membranes are similar. In addition, the similarity between the stria vascularis and the glomeruli could account for why most nephrotoxic medication is ototoxic in nature (Zeigelboim, Mangabeira-Albernaz \& Fukuda, 2001).

The cochlea is dependent on electrolytic stability within the body, which maintains cochlear homeostasis. In order for homeostasis to be maintained, composition of the fluids within the cochlea is essential.
This is achieved through normal kidney functioning. The composition of fluids within the inner ear ensures electromotility of the outer hair cells (OHCs). Endolymph contains a high concentration of potassium $\left(\mathrm{K}^{+}\right)$and low concentrations of sodium $\left(\mathrm{Na}^{+}\right)$and calcium $\left(\mathrm{Ca}^{+}\right)$, while perilymph has high concentrations of $\mathrm{Na}^{+}$and is low in $\mathrm{K}^{+}$. The $\mathrm{K}^{+}$-rich endolymph bathes the apex of the membranous labyrinth while the perilymph bathes the basolateral side (Couloigner, Sterkers \& Ferrary, 2006). $\mathrm{K}^{+}$is essential as it is a major charge carrier associated with transduction of the electrical charges. $\mathrm{Na}^{+}$too is important to the auditory system and the maintenance of $\mathrm{Na}^{+}$levels within the perilymph ensures that the electric currents within the auditory system are transmitted. In this manner, the sodium-potassium pump within the inner ear ensures regulation of these electrolytes.

Any interruption to the electrolytic balances within the body, which occurs in patients with $\mathrm{CKD}$, is likely to result in a disturbance to the fluids of the inner ear, increasing the possibility of auditory dysfunction. Auditory dysfunction is further exacerbated by the presence of concomitant conditions, together with the intake of ototoxic medication. The deterioration of kidney functioning does in fact vary in patients; therefore, a classification system is used in order to stage the patient according to the deterioration of the glomerular filtration rate (GFR), which is described as the 'volume of filtrate produced by both the kidneys per minute' (National Kidney Foundation, 2003). The GFR remains the best estimate for kidney functioning. Arora (2008) describes how the GFR is used to measure kidney functioning as well as to categorise patients into stages of the disease. Table 1 displays the staging of patients.

As the disease progresses, so too does the deterioration in kidney function, ultimately resulting in the patient reaching end stage renal disease (ESRD). It is postulated by the researchers that a similar decline occurs in auditory functioning, which could occur as a result of one or a combination of factors, including the disease itself, concomitant conditions such as hypertension/high blood pressure (HBP) and diabetes, as well as intake of ototoxic medication. These conditions are more likely to impact on cochlear functioning; the literature indicates that cochlear alterations have been observed in patients with diabetes, HBP and CKD (Fukushima, Paparella, Schachern \& Harada, 2005; Sobh et al., 1999). It is common for both HBP and diabetes to be associated with CKD; in fact, HBP is a leading cause of renal dysfunction in 
patients with diabetes (Duck, Prazma, Bennet, \& Pillsburg, 1997). In order for effective monitoring programmes to be implemented by audiologists, these three dimensions must be investigated and understood in the patient with CKD. More importantly, specific monitoring needs to be provided for cochlear functioning.

Pure tone audiometric testing, because of its ability to provide frequency-specific information, as well as its accuracy with quantifying auditory function, can provide valuable clinical data which can guide intervention and management (Roeser, Valente \& Hosford-Dunn, 2000). The only limitation of pure tone audiometric testing is that subclinical hearing loss cannot be identified. Distortion product otoacoustic emissions (DPOAEs) are known to be sensitive to early cochlear dysfunction, thus sensitively identifying a subclinical hearing loss. DPOAEs originate as a result of interaction of two simultaneous stimulus-related excitation patterns on the basilar membrane, which results in the emergence of a third tone at a specific frequency region of the basilar membrane (Kemp, 1986; Withnell, Shaffer \& Talmadge, 2003). DPOAEs are preferred for the evaluation of cochlear functioning in view of their high test-retest reliability (Nikolopoulos et al., 1997) as well as their ability to evaluate a broader frequency range, thereby providing valuable information about the high-frequency range in particular.

It is plausible to assume that any physiological disruption to the fluids within the cochlea caused by $\mathrm{CKD}$, together with the intake of ototoxic medication and the interaction of concomitant conditions, can impact on cochlear functioning, resulting in abnormal DPOAEs as well as abnormal audiometric thresholds. Both DPOAEs and pure tone audiometry can be used to evaluate cochlear functioning. It is useful to investigate how both these tests can provide information on cochlear functioning in patients with CKD, and how additional factors, including drug intake, concomitant conditions and electrolyte levels, effect cochlear functioning. This information becomes valuable in directing audiological management in this population to improve quality of life for patients with CKD.

\section{Methods \\ Objective}

To determine cochlear function in a group of patients with varying stages of CKD through the use of pure tone audiometry and DPOAEs.

\section{Research context}

The study was conducted at a renal unit based at a tertiary hospital in KwaZulu-Natal.

\section{Participants}

The study sample consisted of 50 participants suffering from varying stages of CKD, ranging between the ages of 18 and 45 years (so as to exclude the potential effects of presbycusis), with a mean age of 33.6 years.

Exclusion criteria: Participants were excluded if they presented with:

- Pre-existing hearing loss prior to diagnosis of CKD diagnosed through pure tone audiometric testing

- History of exposure to risk factors of hearing loss, e.g. noise exposure, smoking, consumption of ototoxic medication prior to the CKD diagnosis

- Abnormal middle-ear function, confirmed through assessment of middle-ear functioning

- Family history of hearing loss

- Any vestibular pathology

- HIV/AIDS, cancer or tuberculosis.

\section{Study design}

A quasi-experimental cross-sectional with-in subject design was employed for the study.

\section{Data collection and analysis}

Pure tone audiometric testing and DPOAEs were both conducted in a soundproof room using calibrated equipment. The octave frequency ranges of $250 \mathrm{~Hz}$ to $8000 \mathrm{~Hz}$ were measured for pure tone audiometry and the corresponding geometric frequencies obtained from DPOAE testing. The results were plotted onto an audiogram. Participants who presented with hearing thresholds of $25 \mathrm{~dB}$ HL or less were considered to have normal hearing as indicated by normative data which is displayed in Table 2 (Gelfand, 1997). The 55/65 Vanderbilt norms were implemented to interpret DPOAE data (Biologic Biologic Systems Corp Scout system operating manual, 2002).

Normal cochlear functioning was determined by the distortion product minus the noise floor, being $\geq 6 \mathrm{~dB} \mathrm{nHL}$ (DP-NF=6 dB) (Biologic Scout system operating manual, 2002). The results were plotted onto a DPgram. The results of each audiometric test conducted on each participant were compared for similarity or differences as both tests are known to evaluate auditory functioning differently. Both pure tone audiometric results and DPOAE results for the low- and midfrequency range of $250 \mathrm{~Hz}$ to $3000 \mathrm{~Hz}$ were within normal limits for all participants. This finding implied that there were no significant differences in the two tests' ability to detect normal hearing function in the frequency range of $250 \mathrm{~Hz}$ to $3000 \mathrm{~Hz}$ within the groups studied. Both tests were equally effective in detecting normal hearing function in the low- and mid-frequency region of hearing.

However, significant differences were noted between the tests in the higher-frequency range extending from $4000 \mathrm{~Hz}$ to $8000 \mathrm{~Hz}$. This is highlighted in the ensuing discussion. Pure tone audiometric thresholds of $4000 \mathrm{~Hz}, 6000 \mathrm{~Hz}$, and $8000 \mathrm{~Hz}$ and the corresponding geometric means (GMs) of $3333 \mathrm{~Hz}, 5000 \mathrm{~Hz}$ and $6667 \mathrm{~Hz}$ for DPOAEs were utilised and considered for statistical analysis by applying the McNemar test. The McNemar test involves the use of a two-by-two contingency table to compare the outcome of participants' results on two tests and is a test on matched pairs. The McNemar test allowed for the measurement of the level of agreement of the outcomes for both tests. This means that the test determines whether the proportion of participants responding 'yes' for one test (e.g. pure tone audiometry) is the same as the proportion of pairs responding 'yes' for the other test (e.g. DPOAEs). Table 3 illustrates the McNemar test where the symbol (a) represents the number of participants that had a response 'yes' on both tests. The symbol (b) represents the number of participants that responded 'yes' on the first test and 'no' on the second test. The symbol (c) represents the number who responded 'no' on the first test and 'yes' on the second test, while (d) represents the number who responded 'no' on both tests. In this study (a) represents the cases where the pure tone and DPOAEs test outcomes are both normal, (b) represents the number of participants having normal pure tone audiometric test and abnormal DPOAEs test, while (c) participants have

Table 1. Measurement of kidney functioning and staging

\begin{tabular}{lll}
\hline Stage & Description & $\begin{array}{l}\text { GFR, } \\
\mathbf{~ m l} / \mathbf{m i n} / \mathbf{1 . 7 3 \mathbf { m } ^ { 2 }}\end{array}$ \\
\hline 1 & $\begin{array}{l}\text { Slight kidney damage with normal or } \\
\text { increased filtration }\end{array}$ & $>90$ \\
2 & Mild decrease in kidney function & $60-89$ \\
3 & Moderate decrease in kidney function & $30-59$ \\
4 & Severe decrease in kidney function & $15-29$ \\
5 & $\begin{array}{l}\text { Kidney failure requiring dialysis or } \\
\text { transplantation }\end{array}$ & $<15$ \\
GFR = glomerular filtration rate.
\end{tabular}

Table 2. Categorisation of hearing thresholds

\begin{tabular}{ll}
\hline Categorisation & Range $(\mathbf{d B ~ H L})$ \\
\hline Normal hearing & $0-25$ \\
Mild SNHL & $26-40$ \\
Moderate SNHL & $41-55$ \\
Moderately severe SNHL & $56-70$ \\
Severe SNHL & $71-90$ \\
Profound SNHL & $>90$ \\
SNHL = sensorineural hearing loss &
\end{tabular}


abnormal pure tone and normal DPOAEs. (d) represents the number of participants that have abnormal pure tone and DPOAEs outcomes. To determine which test/s identified cochlear dysfunction, only the frequencies (b) and (c) are considered as these are the cases where the tests give different outcomes while frequencies (a) and (d) provide information on where the tests lead to the same classification outcome.

In addition, electrolytes, urea and creatinine levels were obtained from each patient. Blood results were analysed using one-way analysis of variance, Duncan's multiple range test and Tukey test. Duncan's multiple range test and Tukey test make multiple comparisons of data and are used to detect where differences in means lie. Tukey test examines all pairwise differences and Duncan's multiple range test forms groups of variables with similar means and separates groups on the basis of significantly different means. Therefore, the electrolyte levels, together with the urea and creatinine levels, were evaluated for participants at each stage and the data were compared between each group to determine if the differences in mean values were significant. Concomitant conditions, together with furosemide and other ototoxic drug intakes were entered into cross-tabulation tables. The $p$-value of $<0.05(5 \%)$ was taken as statistically significant, implying that significant differences occurred between the pure tone audiometric thresholds and the DPOAEs, as well as between DPOAE results and blood test results. The approximate $95 \%$ confidence interval was used in this study.

Participants' concomitant conditions including HBP, cardiovascular disease, lupus and diabetes were recorded and included in the analysis of results. Intake of furosemide and other ototoxic drugs such as aminoglycosides was also recorded. All participants underwent pure tone audiometric testing (air and bone), DPOAEs and blood testing. Blood tests were conducted prior to the audiometric examination to determine electrolyte, urea and creatinine levels for each participant. Patients with stage $5 \mathrm{CKD}$ were all receiving haemodialysis; audiometric testing was conducted prior to the dialysis sessions to keep the time of testing standardised.

\section{Ethical considerations}

Ethical clearance was obtained from the Biomedical Ethics Research Committee (BE267/09), University of KwaZulu-Natal. Confidentiality and anonymity were maintained at all times with patients' names being replaced with research numbers. Patients were free to withdraw from the study at any stage and rest periods were given between tests because of the nature of the illness. A nephrologist was consulted at each stage of the study and monitored patients during their testing. Every patient was given an information document, outlining details of hearing loss and hearing conservation.

\section{Reliability and validity}

DPOAE testing was repeated to ensure consistency of responses. Testing was performed in a soundproof room, thereby reducing the presence of background noise. All equipment was calibrated. Patients' blood results and GFR classification were reviewed and verified by an onsite nephrologist.

\section{Results \\ Audiometric results}

\section{Participants in stages 1 and 2 of CKD}

Participants in stages 1 and 2 all presented with normal auditory function with all participants having hearing thresholds of $25 \mathrm{~dB} H \mathrm{HL}$ and less, as well as DPOAE results of $\geq 6 \mathrm{~dB} \mathrm{nHL}$. Therefore, there were no significant differences between both tests $(p>0.0 .5)$.

\section{Participants in stages 3, 4 and 5 of CKD}

In stage 3 , results indicated that there were no significant differences between pure tone audiometry and DPOAEs in detecting normal and abnormal responses across the high-frequency range of $4000 \mathrm{~Hz}$ and $8000 \mathrm{~Hz}$ and the geometric means of $3333 \mathrm{~Hz}$ and $6667 \mathrm{~Hz}$. However, at $6000 \mathrm{~Hz}$ and the geometric mean of $5000 \mathrm{~Hz}$, there was a significant difference $(p<0.01)$, with DPOAEs being more sensitive in detecting abnormal responses than pure tone audiometry. Table 4 displays the results obtained for stage 3 patients.
At $6000 \mathrm{~Hz} / 5000 \mathrm{~Hz}$, both pure tone audiometry and DPOAEs detected $12(60 \%)$ of the 20 observations as normal, however, DPOAEs detected a total of 7 (35\%) of 20 observations as abnormal responses whereas pure tone audiometry detected these as normal responses. Therefore, the levels of agreement between the two tests do differ significantly in the observation of abnormal and normal responses being detected, McNemar chi-squared 7.0; $p<0.01$. This indicates that DPOAEs were more sensitive in detecting early cochlear dysfunction in stage 3 participants.

In stage 4 , there were no significant differences between pure tone audiometry and DPOAEs in detecting abnormal and normal responses across the high-frequency range of $4 \mathrm{kHz}$ and $8 \mathrm{kHz}$ and the geometric means of $3333 \mathrm{~Hz}$ and $6667 \mathrm{~Hz}$. However, at $6000 \mathrm{~Hz}$ and the geometric mean of $5000 \mathrm{~Hz}$, there was a significant difference $(p<0.03)$, with DPOAEs being more sensitive in detecting abnormal responses than pure tone audiometry. Table 5 displays the results obtained for stage 4 patients.

According to the analysis at $6000 \mathrm{~Hz} / 5000 \mathrm{~Hz}$, both pure tone audiometry and DPOAEs could detect normal responses in $8(40 \%)$ of the 20 observations, however, DPOAEs detected a total of $7(35 \%)$ of 20 observations as abnormal responses whereas pure tone audiometry detected these as normal responses. The level of agreement between the two tests does differ significantly in the observation of abnormal and normal responses being detected (McNemar chi-squared 4.50, $p<0.03$. This indicates that DPOAEs were more sensitive in detecting early cochlear dysfunction in stage 4 participants.

In stage 5 , there were no significant differences between pure tone

Table 3. An example illustrating the McNemar test

\begin{tabular}{lll}
\hline & \multicolumn{2}{c}{ DPOAEs } \\
\cline { 2 - 3 } & Yes & No \\
\hline Pure tone audiometry & & \\
Yes & $\mathrm{a}$ & $\mathrm{b}$ \\
No & $\mathrm{c}$ & $\mathrm{d}$ \\
DPOAEs = distortion product otoacoustic. & &
\end{tabular}

Table 4. Cross classification of pure tone thresholds and DPOAEs for stage 3 participants for $6000 \mathrm{~Hz} / 5000 \mathrm{~Hz}$

\begin{tabular}{clll}
\hline & \multicolumn{3}{c}{ DPOAEs, $\boldsymbol{n}$} \\
\cline { 2 - 4 } & Normal & Abnormal & Total, $\boldsymbol{N}$ \\
\hline Pure tones, $\boldsymbol{n}$ & 12 & 7 & 19 \\
Normal & 0 & 1 & 1 \\
Abnormal & 12 & 8 & 20 \\
Total, $\mathbf{N}$ &
\end{tabular}

McNemar chi-squared 7.0000; $p=0.0082$; degrees of freedom: 1 .

DPOAEs $=$ distortion product otoacoustic emissions.

Table 5. Cross classification of pure tone thresholds and DPOAEs for stage four participants for $6000 \mathrm{~Hz} / 5000 \mathrm{~Hz}$

\begin{tabular}{llll}
\hline & \multicolumn{3}{c}{ DPOAEs, $\boldsymbol{n}$} \\
\cline { 2 - 4 } & Normal & Abnormal & Total, $\boldsymbol{N}$ \\
\hline $\begin{array}{l}\text { Pure tones, } \boldsymbol{n} \\
\text { Normal }\end{array}$ & 8 & 7 & 15 \\
Abnormal & 1 & 4 & 5 \\
Total, N & 9 & 11 & 20 \\
McNemar chi-squared 4.5000; $p=0.0339$; degrees of freedom: 1. & \\
DPOAEs = distortion product otoacoustic emissions.
\end{tabular}


audiometry and DPOAEs in detecting abnormal and normal responses at $6000 \mathrm{~Hz}$ and the geometric mean $5000 \mathrm{~Hz}$. However, at $4000 \mathrm{~Hz}$ and $8000 \mathrm{~Hz}$ and at the geometric means of $3333 \mathrm{~Hz}$ and $6667 \mathrm{~Hz}$, there was a significant difference ( $p<0.01$ and $p<0.05$, respectively) between pure tone audiometry and DPOAEs, with DPOAEs being more sensitive in detecting abnormal responses. According to the analysis for $4000 \mathrm{~Hz} / 3333 \mathrm{~Hz}$, both pure tone audiometry and DPOAEs could detect normal responses in $10(50 \%)$ of the 20 observations. DPOAEs detected 8 (40\%) of 20 observations as abnormal responses whereas pure tone audiometry detected them as normal responses. The level of agreement between the two tests does differ significantly in the observation of abnormal and normal responses (McNemar chisquared 5.4; $p<0.01$. This indicates that DPOAEs were more sensitive in detecting early cochlear dysfunction in stage 5 participants. Tables 6 and 7 display the results obtained for stage 5 participants.

According to the analysis at $8000 \mathrm{~Hz} / 6667 \mathrm{~Hz}$, both pure tone audiometry and DPOAEs could equally detect normal responses in $12(60 \%)$ of the 20 observations. DPOAEs detected $6(30 \%)$ of 20 observations as abnormal whereas pure tone audiometry detected these responses as normal. The level of agreement between the two tests does differ significantly in the observation of abnormal and normal responses being detected (McNemar chi-squared 3.57; $p<0.05$.

The overall results of the study indicated a total of $46 \%$ of the participants failed DPOAEs, while presenting with normal behavioural thresholds in the high-frequency range. This implies that almost 50\% of the sample presented with a subclinical hearing loss, which was not identifiable through basic routine hearing testing. Figure 1 displays the overall results obtained for all participants.

\section{Blood results}

According to the analysis of the blood results, no differences were seen for $\mathrm{Na}^{+}$and carbon dioxide $\left(\mathrm{CO}_{2}\right)$ levels between the 5 stages $(p=0.296$ and $p=0.466$, respectively). In terms of $\mathrm{K}^{+}$, a significant difference $(p<0.02)$ was observed between groups particularly in stages 3,4 and 5. These participants presented with higher levels of $\mathrm{K}^{+}$than the participants in the earlier stages. In terms of urea levels, there was a significant difference $(p>0.00)$ between groups, particularly in stages 4 and 5. Participants presented with elevated urea levels that were outside the normative range of $2.1-7.1 \mathrm{mmol} / \mathrm{l}$. Creatinine levels showed a significant difference $(p<0.00)$ as the levels were seen to be elevated in stages 3,4 and 5 with stage 5 patients showing a much greater elevation outside the normative range of $80-115 \mu \mathrm{mol} / \mathrm{l}$.

\section{Concomitant conditions}

Diabetes mellitus, hypertension (HBP) and lupus were recorded as being the most common comorbidities among participants. Other conditions were grouped under 'other' and are reflected in Table 8.

There appeared to be a fairly equal presence of diabetes, lupus or conditions categorised under 'other' in participants across the 5 stages of $\mathrm{CKD}$, although stage 3 participants did present with a fairly higher number of participants with lupus (60\%). In terms of HBP, stage 5 participants had the highest prevalence of HBP with 9/10 participants (90\%) presenting with this condition. There was generally a high prevalence of this condition as opposed to other conditions across the 5 stages.

\section{Ototoxic medication}

The most frequent ototoxic medication recorded was furosemide with at least $50 \%$ of patients taking aminoglycosides as well. It was found that in the later stages of CKD, stages 3,4 and 5, there was an increased

Table 6. Cross classification of pure tone thresholds and DPOAEs for stage 5 participants for $4000 \mathrm{~Hz}$ / $3333 \mathrm{~Hz}$

\begin{tabular}{llll}
\hline & \multicolumn{3}{c}{ DPOAEs, $n$} \\
\cline { 2 - 4 } & Normal & Abnormal & Total, $N$ \\
\hline $\begin{array}{l}\text { Pure tones, } n \\
\text { Normal }\end{array}$ & 10 & 8 & 18 \\
Abnormal & 1 & 1 & 2 \\
Total, N & 11 & 9 & 20 \\
McNemar chi-squared 5.4444; $p=0.0196$; degrees of freedom: 1. & \\
DPOAEs = distortion product otoacoustic emissions &
\end{tabular}

Table 7. Cross classification of pure tone thresholds and DPOAEs for stage five participants for $8000 \mathrm{~Hz}$ / $6667 \mathrm{~Hz}$

\begin{tabular}{llll}
\hline & \multicolumn{3}{c}{ DPOAEs, $\boldsymbol{n}$} \\
\cline { 2 - 4 } & Normal & Abnormal & Total, $\boldsymbol{N}$ \\
\hline $\begin{array}{l}\text { Pure tones, } \boldsymbol{n} \\
\text { Normal }\end{array}$ & 12 & 6 & 18 \\
Abnormal & 1 & 1 & 2 \\
Total, N & 13 & 7 & 20 \\
McNemar chi-squared 3.5714; $p=0.0588$; degrees of freedom: 1. & \\
DPOAEs = distortion product otoacoustic emissions &
\end{tabular}

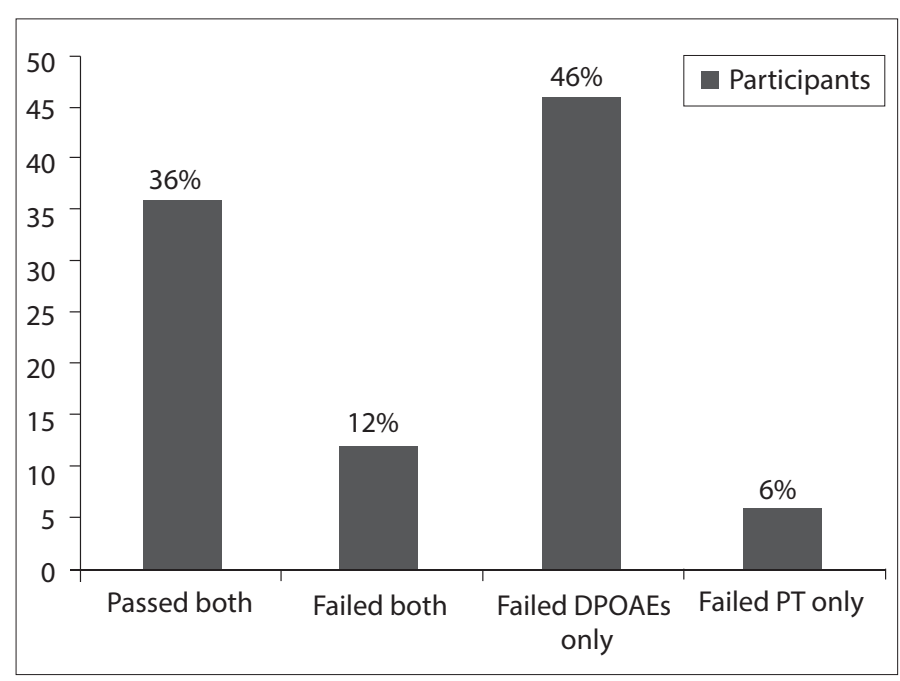

Fig. 1. Overall results of all participants.

Table 8. Cross tabulation of the various concomitant conditions

\begin{tabular}{|c|c|c|c|c|c|c|c|c|c|}
\hline \multirow[b]{2}{*}{ Stage } & \multicolumn{2}{|c|}{ Diabetes } & \multicolumn{2}{|c|}{ High blood pressure } & \multicolumn{2}{|c|}{ Lupus } & \multirow[b]{2}{*}{ Arthritis } & \multirow[b]{2}{*}{ Thyroid } & \multirow[b]{2}{*}{ Other } \\
\hline & No & Yes & No & Yes & No & Yes & & & \\
\hline $1(n=10)$ & 8 & 2 & 3 & 7 & 6 & 4 & 8 & 0 & 1 \\
\hline $2(n=10)$ & 10 & 0 & 4 & 6 & 6 & 4 & 8 & 0 & 1 \\
\hline $3(n=10)$ & 7 & 3 & 2 & 8 & 4 & 6 & 9 & 0 & 1 \\
\hline $4(n=10)$ & 8 & 2 & 3 & 7 & 8 & 2 & 10 & 0 & 0 \\
\hline $5(n=10)$ & 6 & 4 & 1 & 9 & 10 & 0 & 9 & 1 & 0 \\
\hline Total $(N=50)$ & 39 & 11 & 13 & 37 & 34 & 16 & 44 & 1 & 3 \\
\hline
\end{tabular}


number of participants taking furosemide, with the highest number of participants being in stage $5(7 / 10 ; 70 \%)$. Table 9 displays the number of participants on furosemide.

\section{Discussion}

Patients with stages 1 and 2 CKD present with essentially normal kidney functioning and are therefore asymptomatic (Weiner, 2007). It is thus reasonable to assume that in the presence of a normal GFR the systems of the body, including the auditory system, may not be affected or disturbed by the disease at this stage. A possible explanation for this could be related to a process referred to as hyperfiltration, whereby the filtration rate of the remaining nephrons is increased (Gulati, 2010), therefore compensating for the existing damage. It is perhaps also for this reason that patients who are in the initial stages of CKD have almost normal serum creatinine levels. In addition, homeostatic regulation tends to keep $\mathrm{Na}^{+}, \mathrm{K}^{+}, \mathrm{CO}_{2}$, urea and creatinine within the normal ranges which was revealed when the blood results were analysed for stages 1 and 2 .

A significant difference in stage $3(p<0.01)$ and stage $4(p<0.03)$ participants between pure tone thresholds and DPOAE amplitudes was seen at $6000 \mathrm{~Hz}$ and at the corresponding GM frequency of 5 $000 \mathrm{~Hz}$. A significant difference was seen at the high-frequency range of $4000 \mathrm{~Hz}(p<0.01)$ and $8000 \mathrm{~Hz}(p<0.05)$ and the corresponding GM frequency of $3333 \mathrm{~Hz}$ and $6667 \mathrm{~Hz}$ in stage 5 participants, with DPOAEs being significantly more sensitive in identifying the presence of abnormality i.e. cochlear dysfunction. In addition, when the blood test results in group 5 were statistically analysed; it became apparent that the $\mathrm{K}^{+}$, urea and creatinine levels were relatively higher and outside of the normative range when compared with participants in the earlier stages of CKD. It was also evident that when the concomitant conditions were evaluated, participants in stage 5 presented with a higher incidence of HBP $(90 \%)$. With regard to ototoxic medication, furosemide was taken by $70 \%$ of participants in stage 5, with a combined intake of furosemide and aminoglycosides in certain participants. Participants in stages 3, 4 and 5 all presented with abnormal GFRs. It can be hypothesised that patients in advanced stages of CKD, particularly stage 5 patients present with early cochlear dysfunction. This dysfunction could be attributed to one or a combination of factors including elevated electrolyte, urea and creatinine levels, concomitant conditions such as HBP and ototoxic medication which includes furosemide. The glomerulus is not functioning actively; therefore, physiological changes are likely to occur in the body, including changes to the cochlea. These changes are specific to the stria vascularis as a result of the electrolyte imbalance (Thodi et al., 2006). This was confirmed by electrolyte, urea and creatinine levels particularly for stage 5 patients extending outside of the normative ranges. This implies that the sodiumpotassium pump operating within the cochlea could be affected, thus resulting in a disruption of the fluids of the inner ear. The inner ear contains endolymph, which contains specific concentrations of sodium and potassium (Boys Town National Research Hospital, 1989). Endolymph is instrumental in bathing the sensory cells of the inner ear, thus allowing them to function normally (Boys Town National Research Hospital, 1989). Yassin, Badry \& Fatt-Hi (1970) commented on the relationship between hearing loss and electrolyte disturbances in that similar defects in both the kidney and the cochlea occur in respect of transportation of ions. Therefore, it seems reasonable to suggest that an imbalance of electrolytes in the body can lead to a disturbance of inner-ear fluids. An imbalance in electrolyte levels can interfere with the normal functioning of the endolymph, thus inducing cochlear dysfunction. Brownell (1982) suggested that sensory transduction within the hair cells of the cochlea occurs as a result of the exchange of $\mathrm{K}^{+}$ions with $\mathrm{Na}^{+}$ions. The levels between the ratios of $\mathrm{K}^{+} / \mathrm{Na}^{+}$differ between the OHCs and inner hair cells (IHCs). The stria vascularis has been identified as the part responsible for the composition of $\mathrm{K}^{+}$and $\mathrm{Na}^{+}$levels within the endolymph (Anniko, Lim \& Wroblewski, 1984). Therefore, a disruption to the electrolyte balance within the body could result in a disturbance to the normal functioning of the stria vascularis, resulting in OHC dysfunction. Findings of the present study are in agreement with findings of
Table 9. Cross tabulation of furosemide intake across the five stages of chronic kidney disease

\begin{tabular}{lll}
\hline \multirow{2}{*}{ Stage } & \multicolumn{2}{c}{ Furosemide } \\
\cline { 2 - 3 } $1(n=10)$ & No & Yes \\
$2(n=10)$ & 7 & 3 \\
$3(n=10)$ & 4 & 6 \\
$4(n=10)$ & 6 & 4 \\
$5(n=10)$ & 3 & 6 \\
Total $(N=50)$ & 3 & 7 \\
& 24 & 26
\end{tabular}

a study conducted by Nikolopoulos et al. (1997), where auditory functioning in young patients with CKD was investigated.

The discussion has detailed how CKD itself can influence auditory dysfunction. However, diabetes mellitus itself can cause cochlear dysfunction. Histopathological studies of the inner ear of diabetic patients revealed capillary wall thickening in the stria vascularis, decrease in the number of fibres existing in the spiral lamina, organ of corti degeneration and $\mathrm{OHC}$ function reduction (Lisowska, Namystowski, Morawski \& Strojiek, 2001). With regard to HBP, McCormic, Harris, Hartley \& Lassiter (1982) stated that a natriuretic hormone, which is found in the blood stream of patients with HBP could be responsible for cochlear dysfunction in these patients; however, more studies need to be conducted in this area to update the literature. This hormone is thought to inhibit the action of the sodium-potassium pump, thus interfering with the integrity of the stria vascularis. Another factor which could impact on cochlear functioning is the drug intake of such patients which is regarded as ototoxic in nature. According to Zeigelboim et al. (2001), the cochlear duct is thought to be vulnerable to certain diseases and substances such as antibiotics and ototoxic medication. These lead to disorders that appear to impact hearing in the high frequencies. This could possibly support findings in participants in stages 3, 4 and 5 who presented with abnormal DPOAEs in the higher frequencies. Ototoxic medication which includes furosemide can have an effect on ionic gradients between the endolymph and perilymph, resulting in oedema to the epithelium of the stria vascularis (Mudd, 2008). It is further indicated in the literature that furosemide affects the generation of the endocochlear potential (Schmiedt, Lang, Okamura \& Schulte, 2002).

Various factors can contribute to cochlear dysfunction in patients with CKD and a combination and interaction of all of the abovementioned factors could result in reduced cochlear functioning in CKD patients. These changes were detected by DPOAE testing in this study, indicating the presence of a subclinical hearing loss since normal pure tone thresholds were obtained. It is therefore beneficial in the management of such patients to have DPOAE testing as a routine evaluation. Comparisons between patients on different doses and types of ototoxic medication were outside the scope of the present study; however, future studies comparing such data may reveal useful information which can be used to guide patient care.

The findings of this study indicate that participants in the later stages presented with significantly abnormal DPOAEs. They presented with an increase in $\mathrm{K}^{+}$, urea and creatinine levels. These abnormal results could be attributed to CKD itself, or a combination of CKD with concomitant conditions and ototoxic medication.

The cochlea appears to be most affected by CKD, which can translate into a subclinical hearing loss. The findings of the study support the role of the audiologist within the multidisciplinary team, managing patients with CKD. Future research in this area should include the investigation of auditory functioning in stage 5 patients both pre and post dialysis treatment to evaluate changes to the auditory system. 


\section{References}

Anniko, M., Lim, D. \& Wroblewski, R. (1984). Elemental composition of individual cells and tissues in the cochlea. Acta Otolaryngol, 98, 439- 453. doi: $10.3109 / 00016488409107585$

Arora, P. (2008). Chronic renal failure. Retrieved 19 March 2009 from http:/emedicine. medscape.com/article/238798.

Biologic Systems Corp. (2002). AuDX protocol setup: User's manual. Mundelein, IL Biologic Systems Corp.

Boys Town National Research Hospital (1989). Information on hearing loss - genetics and deafness - ear and kidneys: What's the connection? Retrieved 2 February 2009 from deafness - ear and kidneys: What
http:/www.boystownhospital.org.

Brownell, W.E (1982). Cochlear transduction: An integrative model and review. Hear Res 6 , 335-360. doi:10.1016/0378-5955(82)90064-8

Couloigner, V., Sterkers, O. \& Ferrary, E. (2006). What's new in ion transports in the cochlea? European Journal of Physiology, 453, 11-22. doi:10.1007/s00424-006-0103-4

Duck, S. W., Prazma, J., Bennet, S. \& Pillsbury, H. (1997). Interaction between hypertension and diabetes mellitus in the pathogenesis of sensory-neural hearing loss. Laryngoscope 107(12), 1596-1605. doi:10.1097/00005537-199712000-00004

Fukushima, H., Paparella, M. M., Schachern, P. A. \& Harada, T. (2005). The effects of type 1 diabetes mellitus on the cochlear structure and vasculature in human temporal bones. The Registry, 12(1), 1-5. doi:10.1016/j.otohns.2005.05.545

Gelfand, S. A. (1997). Essentials of audiology. New York: Thieme Medical Publishers.

Gulati, S. (2010). Chronic kidney disease. Retrieved 26 March 2010 from http://emedicine. medscape.com.

Kemp, D. T. (1986). Otoacoustic emissions, travelling waves and cochlear mechanisms. Hear Res, 22, 95-104. doi:10.1016/0378-5955(86)90087-0

Lisowska, G., Namystowski, G., Morawski, K. \& Strojiek, K. (2001). Cochlear dysfunction and diabetic microangiopathy. Scand Audiol, 30(52), 199-203. doi:10.1080/010503901300007524

McCormic, G., Harris, D.T., Hartley, C.B \& Lassiter, R. B. H. (1982). Spontaneous genetic hypertension in the rat and its relationship to reduce cochlear potentials: Implications for preservation of human hearing. Proc Natl Acad Sci, USA, 79, 2668. doi:10.1073 pnas.79.8.2668

Mudd PA. (2008). Ototoxicity. Medscape. Retrieved on 10 September 2012 from: http:// emedicine.medscape.com

National Kidney Foundation (2003). K/DOQI Clinical Practice Guidelines for Chronic Kidney Disease: Evaluation, classification and stratification. Am J Kidney Dis, 2(1), S1 266. doi:10.7326/0003-4819-139-2-200307150-00013

Nikolopoulos, T. P., Kandiloros, D. C., Segas, J. V., Nomicos, P. N., Ferekidis, E. A., Michelis, K. E., Apostolopoulos, N. J. \& Adamopoulos, G. K. (1997). Auditory function in young patients with chronic renal failure. Clin Otolaryngol, 22, 222-225. doi:10.1046/j.13652273.1997.00890.x

Roeser, R. J., Valente, M. \& Hosford-Dunn, H. (2000). Audiology diagnosis (2nd ed.). New York: Thieme publishers.

Schmiedt R. A., Lang, H., Okamura, H. \& Schulte B. A. (2002). Effects of furosemide applied chronically to the round window: A model of metabolic presbyacusis. J Neurosci, 22(21), 9643-9650.

Sobh, A. M., El Koussi, M. H. \& Bakr, S. M. (1999). Value of otoacoustic emission in monitoring hearing acuity in chronic renal failure patients. Saudi Journal of Kidney Diseases and Transplantation 2, 144-147.

Thodi, C., Thodis, E., Danielides, V., Pasadakis, P. \& Vargemezis. (2006). Hearing in renal failure. Nephrol Dial Transplant, 21, 3023-3030. doi:10.1093/ndt/gfl472

Weiner, D. E. (2007). Causes and consequences of chronic kidney disease: Implications for managed health care. Journal of Management Care Pharm, 13(3), s1-s9.

Withnell, R. H., Shaffer, L. A. \& Talmadge, C. L. (2003). Generation of DPOAEs in guinea pig. Hear Res, 178, 106-117. doi:10.1016/s0378-5955(03)00064-9

Yassin, A., Badry, A. \& Fatt-Hi, A. (1970). The relationship between electrolyte balance and cochlea disturbances in cases of renal failure. J Laryngol Otol, 84, 429-435. doi:10.1017 s0022215100072030

Zeigelboim, S. B., Mangabeira- Albernaz, P. L. \& Fukuda, Y. (2001). High freq audiometry and chronic renal failure. Acta Otolaryngol, 121, 245-248. doi:10.1080/00016480130004368 